\title{
Ray tracing in anisotropic media with singularities
}

\author{
Václav Vavryčuk* \\ Centro de Pesquisa em Geofisica e Geologia, Instituto de Física, Universidade Federal da Bahia, 40210-340 Salvador, Bahia, Brazil
}

Accepted 2000 November 14. Received 2000 November 10; in original form 2000 July 14

\begin{abstract}
SUMMARY
Singularities often cause trouble in tracing rays in inhomogeneous anisotropic media. The ray-tracing algorithms can break down in the vicinity of the singularities because the medium becomes nearly degenerate and the right-hand sides of the ray-tracing equations yield indefinite expressions. We propose a modified ray-tracing approach, which is numerically stable and yields correct results in all types of singularities and their vicinities. We demonstrate the applicability and efficiency of this approach using various numerical examples. We discuss the possibility of the splitting of a ray when crossing a singularity.
\end{abstract}

Key words: anisotropic media, ray tracing, singularities.

\section{INTRODUCTION}

Singularities are very common in all kinds of anisotropy and often cause difficulties in tracing rays in inhomogeneous anisotropic media. They also cause anomalies in the polarization of a wavefield and in the geometry of wave surfaces (Alshits \& Lothe 1979; Alshits et al. 1985; Crampin \& Yedlin 1981; Grechka \& Obolentseva 1993; Helbig 1994; Vavryčuk 1999). They are defined as the directions where two waves have coincident phase velocities, hence the medium is degenerate in this direction. The waves with a coincident phase velocity are usually $S 1$ and $S 2$ waves, but in principle, the phase velocities of $P$ and $S 1$ waves can also coincide. It is even conceivable that the phase velocities for all three waves coincide in the singularity. We distinguish between kiss, intersection and point singularities. The point singularity is also called the conical point and is probably the most complicated singularity affecting the geometry of rays and the wavefield in the most striking way (Miller \& Musgrave 1956; Burridge 1967; Musgrave 1985; Grechka \& Obolentseva 1993; Rümpker \& Thomson 1994). All these singularities can appear in weakly as well as in strongly anisotropic media. Under strong anisotropy, they usually appear together with so-called triplications, which also complicate the geometry of the wave front. However, triplications do not pose complications in ray-tracing equations, while the singularities can cause breakdowns of the ray-tracing algorithms (Shearer \& Chapman 1989; Červený 2001; Pšenčík \& Dellinger 2001). These breakdowns are connected with numerical instabilities that arise whenever the phase velocity sheets of two waves are close to each other. The medium becomes nearly degenerate

* On leave from: Geophysical Institute, Academy of Sciences of the Czech Republic, Boční II/1401, 14131 Praha 4, Czech Republic. E-mail: vv@ig.cas.cz and the right-hand sides of the ray-tracing equations yield indefinite expressions (Gajewski \& Pšenčík 1990). This results in inaccurate or even erroneous ray tracing, or in a collapse of the algorithm.

In this paper we show that these difficulties can be overcome by formulating a proper ray-tracing strategy. This strategy is based on inspecting the behaviour of the polarization vector of the traced wave whenever the ray approaches the singularity. This strategy can be easily coded and only requires a slight modification of existing ray tracers. Numerical examples are used to demonstrate the applicability and efficiency of our approach. We discuss the possibility of the splitting of the ray in crossing the singularity.

\section{RAY-TRACING EQUATIONS FOR ANISOTROPIC MEDIA}

Ray-tracing equations for anisotropic inhomogeneous media can be expressed in the following form (Červený 1972, eq. 13; Červený et al. 1977, eq. 5.18):

$$
\frac{d x_{i}}{d \tau}=\frac{1}{2} \frac{\partial G}{\partial p_{i}}, \quad \frac{d p_{i}}{d \tau}=-\frac{1}{2} \frac{\partial G}{\partial x_{i}},
$$

where $x_{i}$ are the components of the position vector, $\tau$ is the traveltime, $p_{i}=\partial \tau / \partial x_{i}$ are the components of the slowness vector, $G=G\left(x_{i}, p_{i}\right)$ is the eigenvalue of the Christoffel tensor $\Gamma_{j k}=a_{i j k l} p_{i} p_{l}$, and $a_{i j k l}$ is the density-normalized elasticity tensor. Eigenvalue $G$ is calculated from the Christoffel equation,

$\operatorname{det}\left(\Gamma_{j k}-G \delta_{j k}\right)=0$,

which represents a cubic algebraic equation

$G^{3}-P G^{2}+Q G-R=0$, 
with coefficients

$P=\operatorname{tr}\left(\Gamma_{j k}\right), Q=\operatorname{det}\left(\Gamma_{j k}\right), R=\operatorname{tr}\left(\hat{\Gamma}_{j k}\right)$,

where $\hat{\Gamma}_{j k}$ stands for the matrix of cofactors of $\Gamma_{j k}$.

The Christoffel tensor $\Gamma_{j k}$ has three eigenvalues $G$, which are real-valued and positive, and three eigenvectors g. The eigenvalues correspond to three waves $(P, S 1$ and $S 2)$ propagating in anisotropic media, and the eigenvectors correspond to the polarization vectors of these three waves. Hence, we should determine three systems of rays. If all three eigenvalues of the Christoffel tensor are different for some direction of slowness vector $\mathbf{p}, G^{P} \neq G^{S 1} \neq G^{S 2}$, we speak of the regular direction in anisotropy. If any two eigenvalues coincide for some slowness vector $\mathbf{p}$, we speak of the singular direction in anisotropy. A typical example of the singular direction is the $S$-wave singularity. If two eigenvalues coincide for all directions of $\mathbf{p}$, then the anisotropy degenerates to isotropy.

\section{RAY TRACING IN REGULAR DIRECTIONS}

To evaluate the derivatives $\partial G / \partial p_{i}$ and $\partial G / \partial x_{i}$ on the righthand side of ray-tracing system (1), we can proceed in the following three ways.

(1) We solve eq. (3) analytically and differentiate the solution. However, this approach can be used only for very simple types of anisotropy because in a general case the analytical expressions can be quite complicated and evaluating them is not very effective.

(2) We differentiate eq. (3) using the theorem on implicit functions and express the ray-tracing system in the following form (Červený 1972, eq. 15; Červený et al. 1977, eq. 5.19):

$$
\frac{d x_{i}}{d \tau}=a_{i j k l} p_{l} \frac{\hat{D}_{j k}}{\hat{D}}, \quad \frac{d p_{i}}{d \tau}=-\frac{1}{2} \frac{\partial a_{j k l n}}{\partial x_{i}} p_{k} p_{n} \frac{\hat{D}_{j l}}{\hat{D}}
$$

where $\hat{D}_{j k}$ is the matrix of cofactors of $D_{j k}=\Gamma_{j k}-\mathrm{G} \delta_{j k}$,

$\hat{D}_{11}=\left(\Gamma_{22}-G\right)\left(\Gamma_{33}-G\right)-\Gamma_{23}^{2}$,

$\hat{D}_{12}=\hat{D}_{21}=\Gamma_{13} \Gamma_{23}-\Gamma_{12}\left(\Gamma_{33}-G\right)$,

$\hat{D}_{22}=\left(\Gamma_{11}-G\right)\left(\Gamma_{33}-G\right)-\Gamma_{13}^{2}$,

$\hat{D}_{13}=\hat{D}_{31}=\Gamma_{12} \Gamma_{23}-\Gamma_{13}\left(\Gamma_{22}-G\right)$,

$\hat{D}_{33}=\left(\Gamma_{11}-G\right)\left(\Gamma_{22}-G\right)-\Gamma_{12}^{2}$,

$\hat{D}_{23}=\hat{D}_{32}=\Gamma_{12} \Gamma_{13}-\Gamma_{23}\left(\Gamma_{11}-G\right)$,

where $G=1$ and $\hat{D}$ is defined as

$\hat{D}=\operatorname{tr}\left(\hat{D}_{j k}\right)=\hat{D}_{11}+\hat{D}_{22}+\hat{D}_{33}$.

(3) Taking into account that matrix $\hat{D}_{j k}$ in eqs (5) can be expressed in terms of eigenvectors $g_{k}$ of the Christoffel tensor $\Gamma_{j k}$ as

$\frac{\hat{D}_{j k}}{\hat{D}}=g_{j} g_{k}$,

we obtain the following form of the ray-tracing system (Babich 1961; Červený \& Firbas 1984; Gajewski \& Pšenčík
1987; Shearer \& Chapman 1989, eq. 6):

$\frac{d x_{i}}{d \tau}=a_{i j k l} p_{l} g_{j} g_{k}, \quad \frac{d p_{i}}{d \tau}=-\frac{1}{2} \frac{\partial a_{j k l n}}{\partial x_{i}} p_{k} p_{n} g_{j} g_{l}$.

\section{RAY TRACING IN SINGULAR DIRECTIONS}

If we calculate the rays for waves with coincident eigenvalues, we face difficulties. Since the Christoffel tensor is degenerate, the denominator and nominator terms in eqs (5) become zero and the right-hand sides of these equations yield indefinite expressions $0 / 0$. This produces a numerical instability and causes trouble in ray-tracing problems. The instability arises not only when two eigenvalues coincide, but also whenever they are very close to each other (Shearer \& Chapman 1989; Červený 2001; Pšenčík \& Dellinger 2001). Therefore, eqs (5) are not very appropriate for tracing rays in media with singularities and we shall no longer consider them.

We can avoid the difficulties mentioned by using ray-tracing system (7), which produces no breakdowns in singularities. However, this system produces other difficulties. These arise when we calculate the polarization vectors of traced waves, which are required to evaluate the right-hand sides of the raytracing system. In regular directions, the polarization vectors are uniquely determined as eigenvectors of the Christoffel tensor. In singular directions, however, the eigenvectors for degenerated waves are not unique and thus we have to specify the rules for determining the proper polarization vectors. Hereinafter, we shall attempt to establish such rules for several particular types of singularity.

\subsection{Isotropy and very weak anisotropy}

Interestingly, we meet no difficulties in using eqs (7) to trace the rays of $S$ waves in isotropic media. In isotropy, the right-hand sides of eqs (7) are not sensitive to the actual choice of the $S$-wave polarization vector. Hence, any polarization vector perpendicular to the slowness vector is appropriate for evaluating them and transforms (7) into the standard raytracing system for $S$ waves under isotropy (Červený et al. 1977, eq. 3.2),

$\frac{d x_{i}}{d \tau}=\beta^{2} p_{i}, \quad \frac{d p_{i}}{d \tau}=-\frac{1}{\beta} \frac{\partial \beta}{\partial x_{i}}$,

where $\beta$ is the $S$-wave velocity.

Obviously, a similar situation to isotropy appears in tracing the rays of $S$ waves in very weakly anisotropic media. In this case, the slowness sheets of the $S$ wave almost coincide in all directions. The Christoffel tensor is nearly degenerate, and we can accurately determine only the plane of the $S$-wave polarization. The actual direction of the $S$-wave polarization vectors in this plane is generally determined with low accuracy. Fortunately, the low accuracy of the polarization vectors has no effect on the correct tracing of rays for $S$ waves because of the low sensitivity of ray-tracing equations (7) to the choice of the polarization vector in the $S$-wave polarization plane (see Appendix A). Hence, the rays in isotropic and very weakly anisotropic media can be traced by eqs (7) without any difficulty. 
Of course, ray tracing equations (8) are more effective than eqs (7) when tracing rays in isotropic media. Likewise, ray tracing based on the perturbation approach (Farra 1989; Nowack \& Pšenčík 1991) is more effective than eqs (7) in the case of very weakly anisotropic media.

\subsection{Kiss singularity}

The next type of singularity to be inspected is the kiss singularity. The kiss singularity is defined as the direction in which two $S$-wave slowness surfaces touch tangentially at an isolated point (see Fig. 1). Consequently, $S$-wave fronts are also tangential at the kiss singularity, and the group velocities for the both $S$ waves coincide, being equal to the phase velocity and parallel to the slowness vector at this point. In the vicinity of the singularity, the polarization of $S$ waves behaves anomalously (see Fig. 1), and at the singularity the polarization vectors are not defined uniquely. Nevertheless, this singularity does not pose serious difficulties to ray-tracing system (7). Similarly to isotropy, the right-hand sides of eqs (7) are insensitive to the actual choice of the $S$-wave polarization vector in the singularity. If we insert any polarization vector perpendicular to the slowness vector, then ray-tracing equations (7) take the following simple form in the singularity:

$\frac{d x_{i}}{d \tau}=c^{2} p_{i}, \quad \frac{d p_{i}}{d \tau}=-\frac{1}{c} \frac{\partial c}{\partial x_{i}}$,

where $c$ is the phase velocity of the $S$ waves along the axis of symmetry (the same for both $S$ waves). Since the ray-tracing system is completely independent of the polarization vector of the $S$ wave in the singularity, we can also expect this system to be weakly sensitive to variations of the $S$-wave polarization vector in the close vicinity of the singularity. This property can be proved in a similar way as in Appendix A for $S$ waves in weakly anisotropic media. Therefore, the possibility of the strange or incorrect behaviour of rays due to $S$-wave polarization anomalies near the kiss singularity can be excluded.

\subsection{Intersection singularity}

Difficulties can arise, however, in tracing rays in the vicinity of an intersection singularity. The intersection singularity typically occurs in transversely isotropic (TI) media, where the slowness (or phase velocity) surfaces of $S$ waves ( $S H$ and $S V$ waves) can intersect along a line (see Fig. 2). This singular line in the slowness surface splits into two different lines in the wave surface (Fig. 2, upper right plot). For the singular line, the right-hand sides of eqs (7) depend on the choice of the $S$-wave polarization vector. Hence, this choice has a significant impact on the form of the rays calculated. However, it is not very complicated to find a proper polarization vector of the traced wave in the singularity. For tracing rays of the $S H$ wave in the singularity, we simply use the $S H$-wave polarization, and for the $S V$ wave we use the $S V$-wave polarization (Fig. 2, lower plots). This has been recognized and successfully applied by Shearer \& Chapman (1989), who used analytical formulae for polarization vectors of the $S V$ and $S H$ waves when tracing rays in transverse isotropy. These analytical formulae (Shearer \& Chapman 1989, eqs A8 and A9) guarantee a smooth change of the polarization vector along a ray as the ray passes the singularity.

\section{howness su ace}
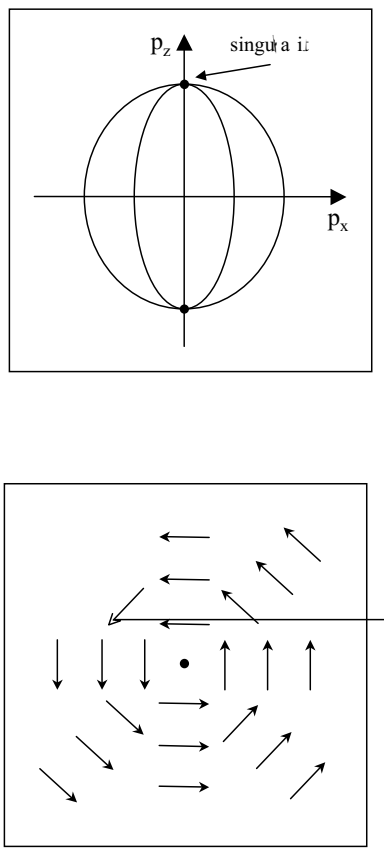

Wave su ace

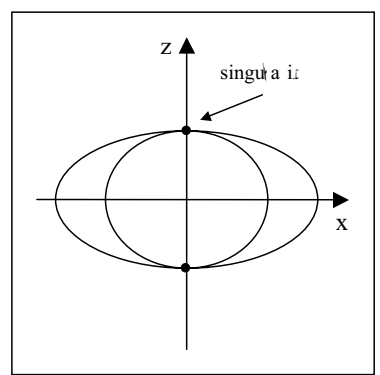


If we evaluate the polarization vector numerically as the eigenvector of the Christoffel tensor, we have no unambiguous rule for calculating the polarization vector in the singularity. However, the numerical approach should follow a similar rule to the analytical approach: when a ray crosses the singularity, the change of the polarization vector along the ray must be smooth. This can be approximately realized by replacing the polarization vector in the singularity by the polarization vector at the point of a ray just before the singularity.

\subsection{Conical point}

The most complicated singularity in anisotropy is a conical point. The conical point (or the point singularity) is defined as the direction, in which two slowness sheets touch through the vertices of cone-shaped surfaces (see Fig. 3). Hence, in the vicinity of this singularity both the slowness sheets look like two identical cones placed tip to tip (Crampin \& Yedlin 1981; Rümpker \& Thomson 1994). The singular point in the slowness sheet (point $\mathrm{P}$ in Fig. 3) generates a singular line in the wave sheet. Hence, all points at the singular line of the wave sheet have the same slowness vector. In other words, a cone of acceptable rays corresponds to one slowness vector at the singular point. Vice versa, wave sheets can also form a conical point (point Q in Fig. 3). This point generates a line of points in the slowness sheet that are associated with the same ray direction.

Despite the complexity of the geometry of the conical point, we have to point out that the behaviour of polarization vectors near the singular line in the wave sheet is surprisingly simple. The polarization vectors behave regularly on both sides of

howness su ace
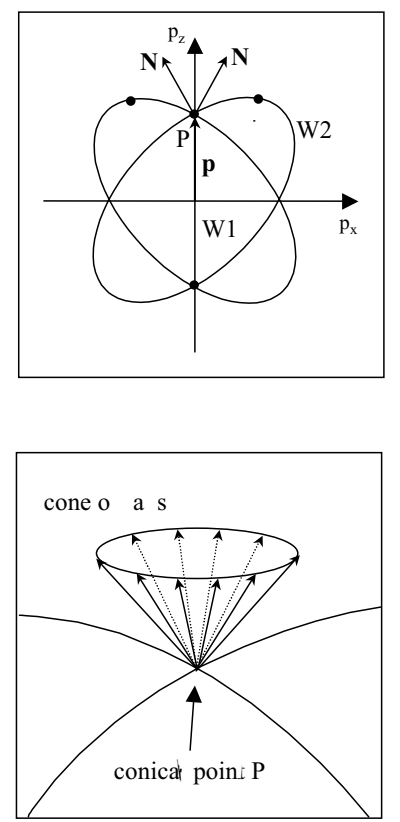

Figure 3. Conical point in Payton's transverse isotropy (Payton 1992). Upper plots show vertical sections of the slowness and wave surfaces of the $W 1$ and $W 2$ waves. Lower plots show a 3-D sketch of the cone of rays generated at the singularity (left-hand plot) and the form of the wave surface near the singular line (right-hand plot). $\mathrm{P}$ denotes the singularity, Q is the conical point at the wave surface. For the meaning of the other quantities, see the caption to Fig. 2. the singular line, and the polarization pattern is similar to that for the intersection singularity (see Fig. 4). Hence, we can assign the proper polarization of the wave at the singular line in the same way as for the intersection singularity: we require a smooth change of polarization along the ray when the ray crosses the singularity.

Note that the order of waves is interchanged when the ray crosses the singularity: the faster wave in front of the singularity becomes the slower wave beyond the singularity, and vice versa.

\section{GENERAL STRATEGY FOR RAY T R A C I N G}

In the previous section we discussed the behaviour of the raytracing equations for several particular types of singularity. Now we generalize these results and draw up a unified strategy valid for tracing rays in any kind of singularity and its vicinity. In this strategy, no information about the type of singularity will be required.

\subsection{Procedure}

\subsubsection{The ray reaches a regular point}

The polarization vector of the traced wave is calculated as the eigenvector of the Christoffel tensor. From the three eigenvectors calculated, we choose the eigenvector that guarantees a smooth change of the polarization vector from the previous to the current point of the ray. We emphasize that it is not sufficient to retain the type of wave $(P, S 1$ or $S 2)$ in the raytracing procedure. Since a singularity can appear between the previous and the current point of the traced ray, the type of the traced wave may change.

Determining the proper polarization vector, we can evaluate the right-hand sides of ray-tracing equations (7) and trace the ray to the next point.

\section{W1-W2 poł a ization}

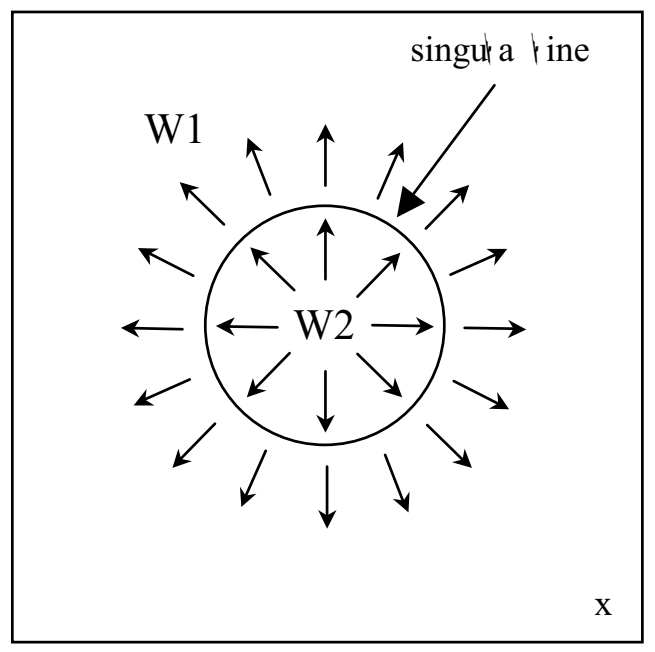

Figure 4. The horizontal projection of the $W 1$ - and $W 2$-wave polarization vectors near the singular line at the wave surface in Payton's transverse isotropy. 


\subsubsection{The ray touches a singularity}

The polarization vector of the traced wave is not determined by the Christoffel equation uniquely. Therefore, we calculate the polarization vector as the limit of polarization vectors at points of the ray just before the ray touches the singularity,

$\mathbf{g}^{+}\left(\tau_{\mathbf{S}}\right)=\lim _{\tau \rightarrow \tau_{\mathrm{S}}^{+}} \mathbf{g}(\tau)$

where $\tau$ is the traveltime along the ray and $\tau_{\mathrm{S}}$ is the traveltime at the singularity. Inserting polarization vector $\mathbf{g}^{+}\left(\tau_{\mathbf{S}}\right)$ into the ray-tracing equations we move forward to the next point of the ray.

\subsubsection{The ray leaves the singularity}

Here, we assume two alternatives.

(i) The next point beyond the singularity is regular. In this case, the polarization vectors of the waves degenerated in the singularity are now determined in the standard way by the Christoffel equation. However, we have to check whether or not the ray becomes split in the singularity. To identify this case, we have to determine the polarization vectors of the waves that degenerated in the singularity. We then calculate the limit of polarization vectors along the ray when going backwards to the singularity,

$\mathbf{g}^{-}\left(\tau_{\mathbf{S}}\right)=\lim _{\tau \rightarrow \tau_{\mathbf{S}}^{-}} \mathbf{g}(\tau)$.

If the polarization vector of one of the degenerated waves beyond the singularity matches continuously the polarization of the wave in front of the singularity,

$\mathbf{g}^{-}\left(\tau_{\mathbf{S}}\right)=\mathbf{g}^{+}\left(\tau_{\mathbf{S}}\right)$,

the ray does not split. We then trace only the ray for this particular wave (see Fig. 5a). In the other case, we have to trace the rays for all waves degenerated in the singularity (see Fig. 5b).

(ii) The next point beyond the singularity is again singular. We consider two cases when this situation occurs. First, when the ray touches the singularity the wave starts to propagate in a homogeneous anisotropic medium. Since the ray becomes a straight line, the wave cannot leave the singularity until an inhomogeneity occurs. Second, the ray plunges into a locally isotropic (homogeneous or inhomogeneous) region. In both the cases we can apply the polarization vector determined at the previous singular point to trace the ray to the next point. In isotropy, we can also switch to simpler ray-tracing system (8) designed for this medium.

\subsection{Numerical implementation}

To apply the above procedure we have to specify how to realize numerically the limits of the polarization vector in the singularity in eqs (10) and (11). Since the ray is calculated at discrete points only, we simply associate limit $\mathbf{g}^{+}\left(\tau_{\mathbf{S}}\right)$ with the polarization vector at the last point on a ray before the singularity. Similarly, we associate limit $\mathbf{g}^{-}\left(\tau_{\mathrm{S}}\right)$ with the polarization vector at the first point on a ray after the singularity. a) $g^{+}\left(\tau_{S}\right)=g^{-}\left(\tau_{S}\right)$

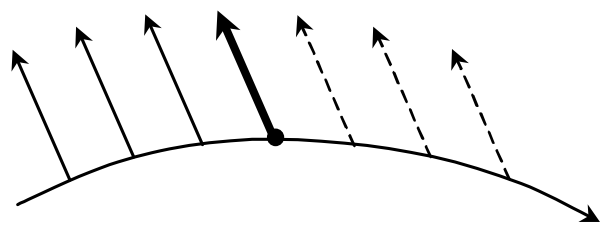

a

b)

$$
g^{+}\left(\tau_{S}\right) \neq g^{-}\left(\tau_{S}\right)
$$

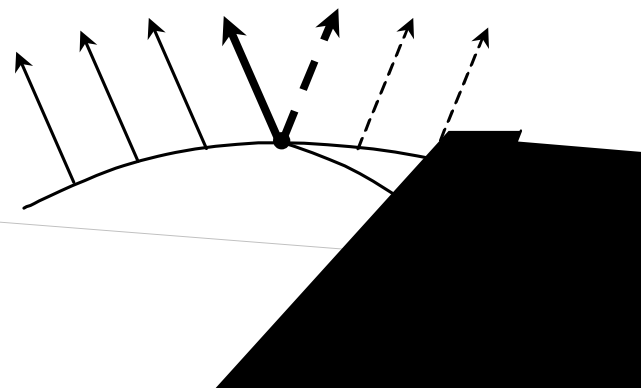

Obviously, to achieve the required accuracy, the coverage of a ray by discrete points must be sufficiently dense in the vicinity of the singularity. Also, in calculating polarization vectors we have to apply an accurate procedure for calculation of the eigenvectors of nearly degenerate matrices.

\section{NUMERICAL EXAMPLES I: SPLITTING OF RAYS}

In this section we apply the proposed ray-tracing strategy to two models of the medium. In both models, a singularity occurs along a plane interface, which separates media with different types of anisotropy. In the first model, the interface separates isotropic and anisotropic layers; in the second model, the interface separates two anisotropic layers. The velocities are continuous functions across the interface, hence the interface generates no scattered waves. Nevertheless, we shall show that all rays incident at the interface split into two different rays.

\subsection{Isotropy/anisotropy transition}

The model consists of two layers: the upper layer is transversely isotropic with a vertical axis of symmetry (VTI layer) and the lower layer is isotropic. Both layers are vertically inhomogeneous. The interface is at a depth of $4 \mathrm{~km}$. The point source is situated in the isotropic layer at a depth of $8 \mathrm{~km}$. The $P$-wave velocities at the source and at the interface are $\alpha=5.5$ and 
$4.5 \mathrm{~km} \mathrm{~s}^{-1}$, respectively. The $S$-wave velocity in the isotropic layer is $\beta=\alpha \sqrt{3}$. The $P$ - and $S$-wave velocities in the isotropic layer increase linearly with depth. The VTI in the upper layer is defined by the density-normalized elastic parameters as follows: $a_{11}=a_{22}=a_{33}, a_{13}=a_{23}=a_{11}-2 a_{44}, a_{44}=a_{55}, a_{66}=a_{44}+2 \varepsilon_{3}$, $a_{12}=a_{11}-2 a_{66}$. At the interface the values of these parameters are $a_{11}=20.25, a_{44}=a_{11} / 3$ and $\varepsilon_{3}=0$, and at the surface, $a_{11}=12.25, a_{44}=a_{11} / 3$ and $\varepsilon_{3}=1$. The anisotropy is controlled by anisotropy parameter $\varepsilon_{3}$ (see Vavryčuk 1997). This parameter is zero at the interface and linearly increases towards the surface. Hence, the medium is isotropic at the interface and the anisotropy gradually increases towards the surface. The $P$ and $S V$ waves have directionally independent velocities $\alpha=\sqrt{a_{11}}$, $\beta=\sqrt{a_{44}}$, which linearly increase with depth. For the $S$-wave velocities at the surface see Fig. 6. The rays of the $S$ wave are shot from the point source in the angle interval $\theta \in\left\langle 0^{\circ}, 90^{\circ}\right\rangle$ in steps of $10^{\circ}$.

Since the transition between the isotropic and the VTI layers is smooth, no scattered waves are generated at the interface. Nevertheless, the rays split at the interface (see Fig. 6, lower plot) because the interface separates the singular and regular anisotropic regions. Since the anisotropy is VTI, the $S$ wave splits into $S V$ and $S H$ waves. The splitting of the $S V$ and $S H$ rays is smooth because the tangents of the split rays coincide at the interface. The deviation between the rays of split waves increases with increasing anisotropy of the VTI layer. If the source generates the $S$ wave with the polarization of the $S V$ or $S H$ wave only, no splitting occurs at the interface.

\subsection{Anisotropy/anisotropy transition}

This model consists of two transversely isotropic layers: the axis of symmetry in the upper layer is vertical (VTI), while in the lower layer it is horizontal (HTI) and deviates from the $x$-axis by $45^{\circ}$. The transverse isotropy of the HTI layer is essentially the same as for the VTI layer. The difference is only in the orientation of the axis of symmetry. At the source the parameters of HTI in the local coordinate system, whose symmetry axis is parallel to the $z$-axis, are $a_{11}=30.25, a_{44}=a_{11} / 3$ and $\varepsilon_{3}=1$, and at the interface, $a_{11}=20.25, a_{44}=a_{11} / 3$ and $\varepsilon_{3}=0$. The velocities of the $P$ and $S R$ waves are $\alpha=\sqrt{a_{11}}$, $\beta=\sqrt{a_{44}}$; they increase linearly with depth. The parameters of the VTI layer are the same as for the isotropy/VTI model. Also, the positions of the source and of the interface are similar to the isotropy/VTI model. Anisotropy parameter $\varepsilon_{3}$ linearly decreases in the HTI layer from unity at the source depth to zero at the interface. Hence, both HTI and VTI layers are in fact isotropic at the interface. The strongest anisotropy is observed at the depth of the source and at the surface, but the anisotropies have different orientations. The rays of the $S$ waves are shot from the point source in the angle interval $\theta \in\left\langle 0^{\circ}, 90^{\circ}\right\rangle$ in steps of $10^{\circ}$, where $\theta$ is the angle between the slowness vector and the vertical axis.

Fig. 7 shows the results of the ray tracing of two $S$ waves (denoted $S R$ and $S P$ waves) generated at the source. Similarly as in the isotropy/VTI model, the interface represents the singular region in the HTI/VTI model. Since the orientation of anisotropy

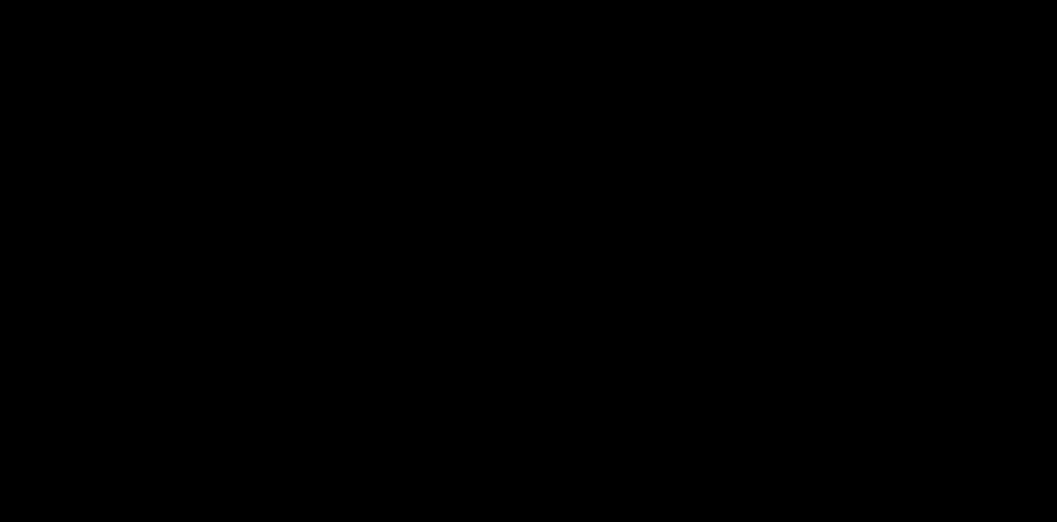




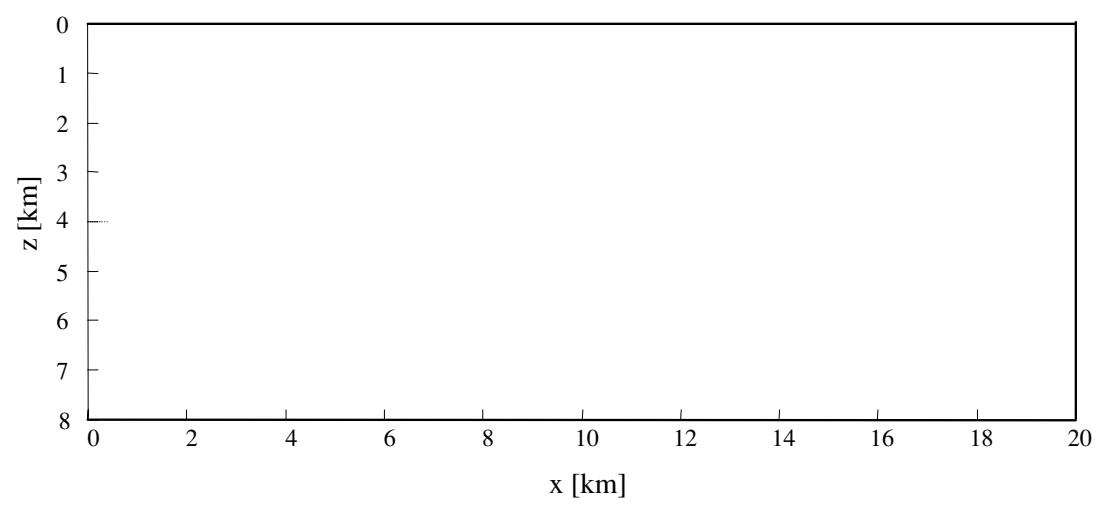

is different on both sides of the singularity, the rays of each $S$ wave split at the singularity. Fig. 7 shows the splitting of both $S R$ and $S P$ waves separately (upper and lower plots).

\section{NUMERICAL EXAMPLES II: FALSE BENDING OF RAYS}

In this section we apply the ray-tracing strategy to vertically inhomogeneous media containing the intersection singularity and the conical point. We demonstrate how important it is to determine the polarization vectors of traced waves in the singularity and in its vicinity properly. We show that neglecting the rules discussed in the previous section can lead to false ray tracing. False ray tracing is reflected in an abrupt change of the ray direction in the singularity. This false bending of rays can be easily corrected when calculating correct polarization vectors at and beyond the singularity.

\subsection{Intersection singularity}

In tracing rays near the intersection singularity we applied a model similar to 'Model 4' introduced and studied by Shearer \& Chapman (1989). This model is extremely anisotropic. Our model displays the same type of anisotropy, but instead of the horizontal axis of symmetry, we use the vertical axis of symmetry. Hence, the elastic parameters of the medium are $a_{11}=a_{22}=20.16, a_{33}=19.63, a_{44}=a_{55}=3.48, a_{66}=6.38$, $a_{13}=a_{23}=7.26, a_{12}=a_{11}-2 a_{66}$. These parameters refer to a source depth of $z_{0}=8 \mathrm{~km}$. The elastic parameters at other depths were calculated as

$a_{k l}=a_{k l}\left(z_{0}\right)\left[1+\varepsilon\left(z-z_{0}\right)\right]^{2}$,

where $\varepsilon=0.04 \mathrm{~km}^{-1}$ is the normalized velocity gradient in the medium. The intersection singularity is in the direction defined by the slowness vector with angle $\theta=58.59^{\circ}$. The rays of the $S$ waves were shot in the $x-z$ plane in the following angular intervals: $\theta \in\left\langle 50^{\circ}, 82^{\circ}\right\rangle$ in steps of $2^{\circ}$ for the slower $S$ wave, and $\theta \in\left\langle 50^{\circ}, 86^{\circ}\right\rangle$ in steps of $2^{\circ}$ for the faster $S$ wave, where $\theta$ is the deviation of the slowness vector from the vertical axis.

Fig. 8 shows sections of the phase and group velocities at the source and the results of the ray tracing. The intersection singularity at the phase surface is split into two singular lines at the wave surface. Moreover, one of the singular lines coincides with the edge line due to triplication. Although the geometry of the wave surface looks complicated, correct ray tracing does not pose serious difficulties. The only problem arises in the singularity, where we require a continuous change of the direction of the polarization vector of the traced wave along the ray. This requirement is important because the order of waves is interchanged in the singularity: the slower wave becomes the faster and the faster wave becomes the slower. If we neglect this fact and assume that the wave should be faster (or slower) 
Phase velocity

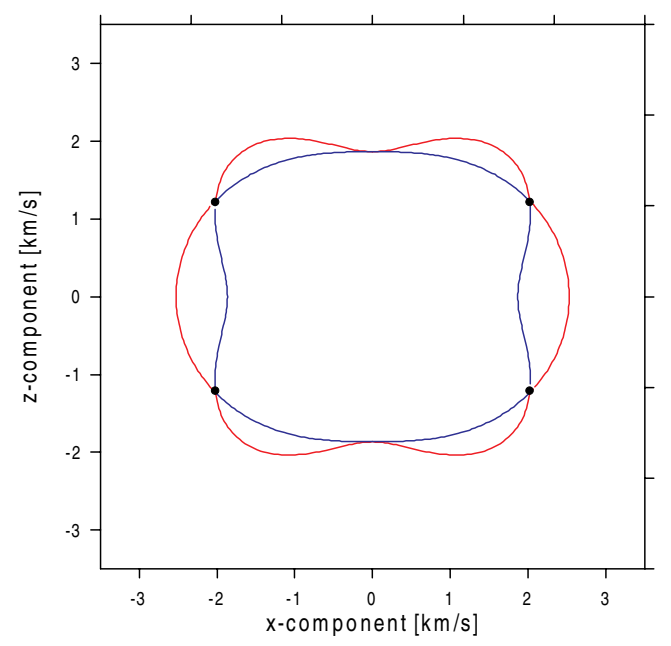

False bending rays

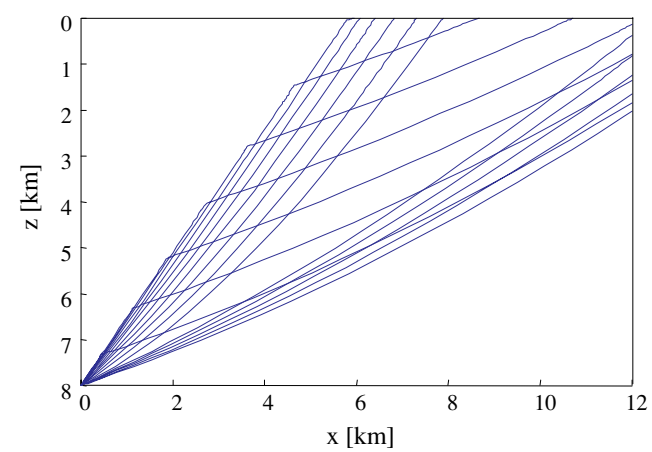

Correct rays

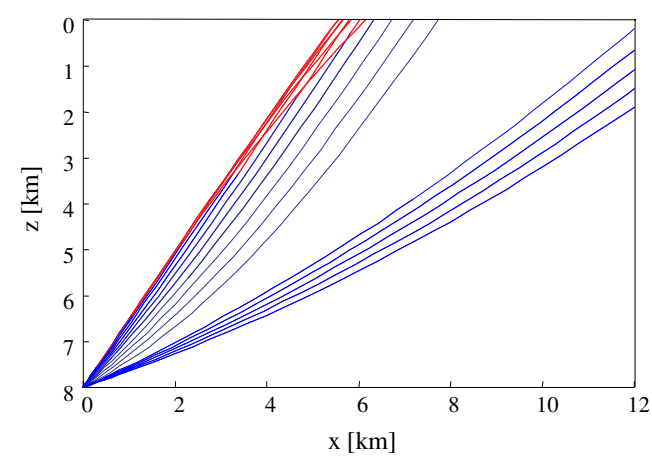

along the entire ray, we obtain spurious results. The change of the polarization vector is discontinuous in the singularity and we observe a false abrupt change of the direction of the ray (Fig. 8, middle plots). Obviously, correct ray tracing (Fig. 8, lower plots) yields smooth curved rays, as is expected in smoothly varying inhomogeneous media.

\subsection{Conical point}

In tracing rays near the conical point, we use a medium displaying cubic anisotropy. The cubic anisotropy is defined by the following elastic parameters: $a_{11}=a_{22}=a_{33}, a_{44}=a_{55}=a_{66}$,
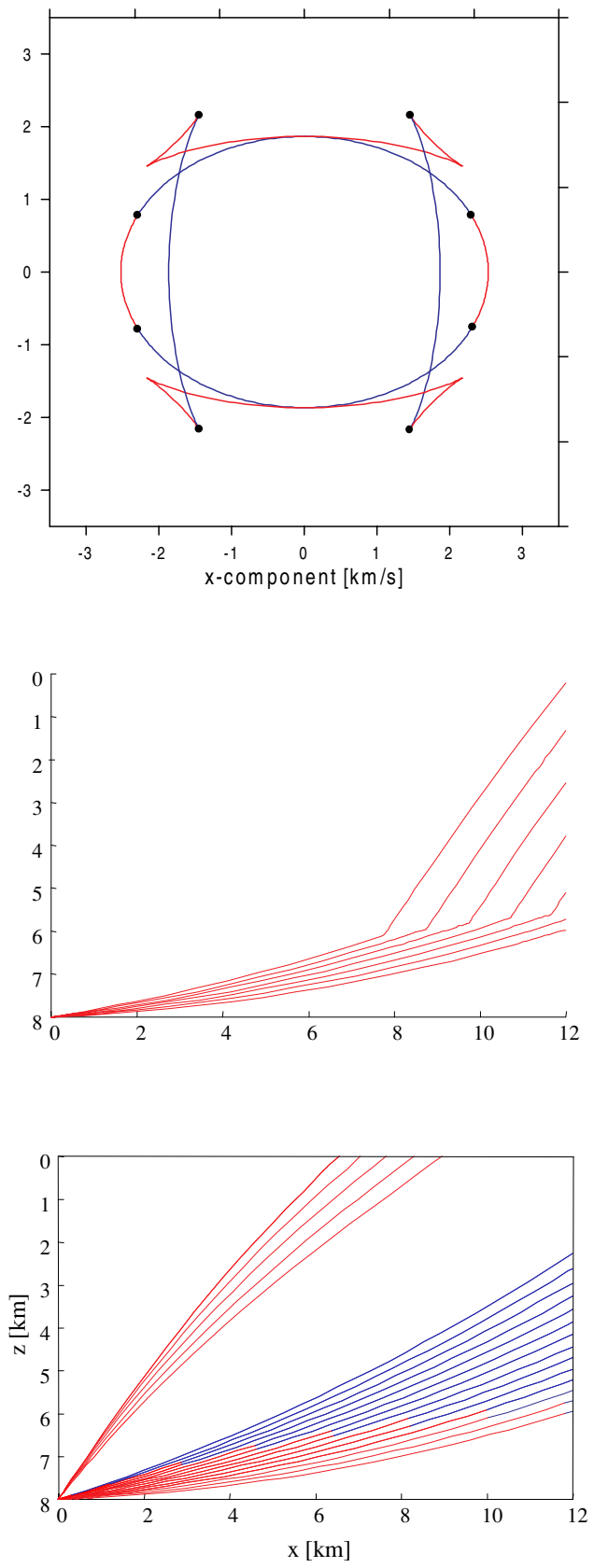

$a_{12}=a_{13}=a_{23}=a_{11}-2 a_{44}+\delta$, where $\delta$ is the parameter measuring the strength of anisotropy. We adopted the following values for the elastic parameters at the source: $a_{11}=6.25, a_{44}=a_{11} / 3$, $\delta=2.00$. The elastic parameters at other depths are provided by eq. (13) with a value for the velocity gradient of $\varepsilon=0.01 \mathrm{~km}^{-1}$. Similarly to the previous models, the source is situated at a depth of $8 \mathrm{~km}$. The conical point in the cubic anisotropy is in the direction defined by the slowness vector with the spherical angles $\phi=45^{\circ}$ and $\theta=54.74^{\circ}$. This implies that the conical point does not lie in the $x-z$ plane. Therefore, the rays of the $S$ waves are shot in the plane, which deviates from the $x-z$ plane by angle $\phi=45^{\circ}$ in the following ranges of angles $\theta: \theta \in\left\langle 44^{\circ}, 69^{\circ}\right\rangle$ in steps of $1^{\circ}$ 
for the faster $S$ wave leaving the source and $\theta \in\left\langle 55^{\circ}, 70^{\circ}\right\rangle$ in steps of $1^{\circ}$ for the slower $S$ wave leaving the source. Angle $\theta$ is the deviation of the slowness vector from the vertical axis.

The sections of the phase and group velocities at the source and the results of the ray tracing are shown in Fig. 9. Neither the phase velocities nor the group velocities are axially symmetric in cubic anisotropy like in transverse isotropy (see Fig. 8). Therefore, the four points in the phase velocity section really represent four point singularities. The eight points in the group velocity section represent a section of four singular circles (for the geometry of the singular line, see Fig. 3). Although the geometry of the conical point is more complicated than the

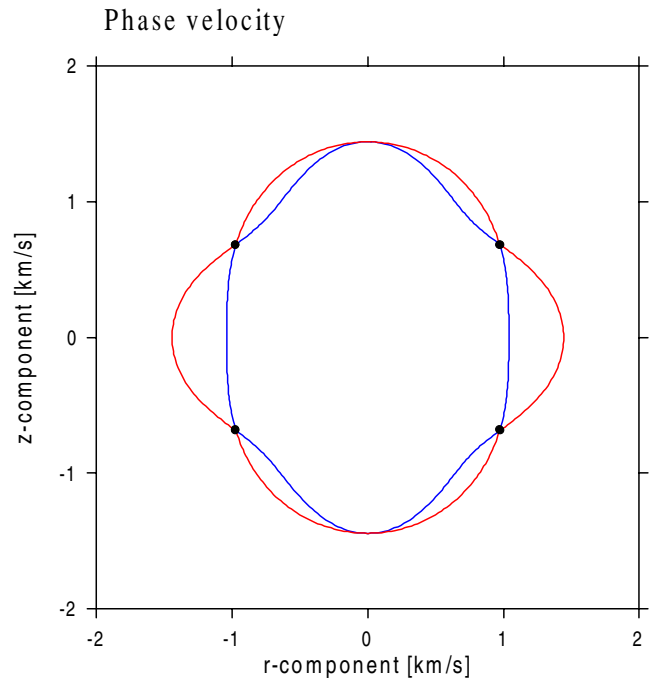

False bending rays

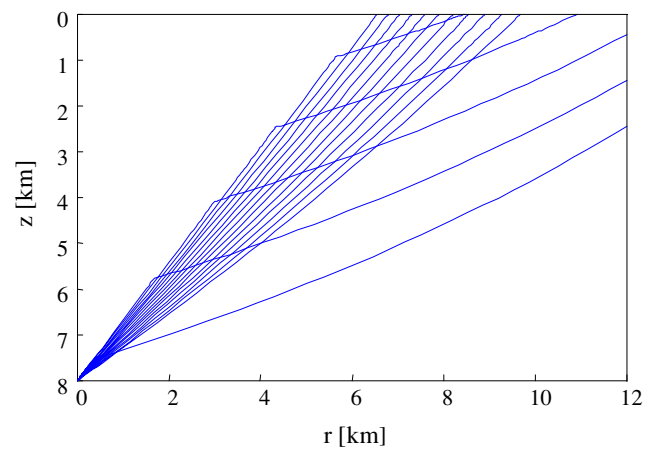

Correct rays

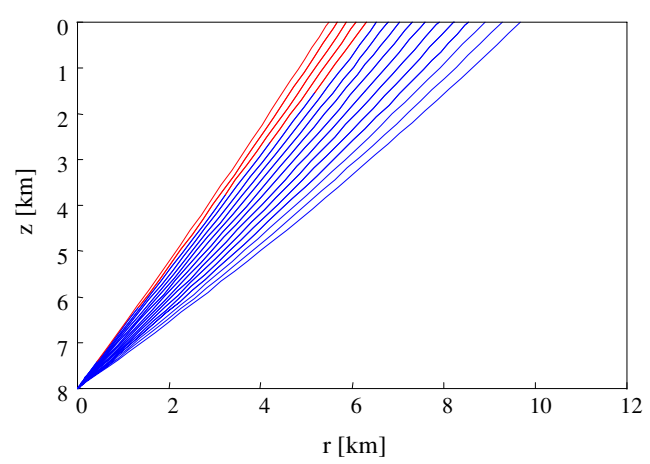

geometry of the intersection singularity, the ray-tracing results are essentially the same in both types of singularity. If we do not take into account the fact that the polarization vectors must be continuous along a ray, the rays abruptly bend in the singularity. If we interchange the type of the traced wave in the singularity ( $S 1$ to $S 2$ and $S 2$ to $S 1$ ) because of the continuity of the polarization vector of the traced wave, the rays are smooth and the abrupt ray bending disappears. The situation significantly changes, however, if rays come very near to the singularity but do not cross it. Fig. 10 shows ray tracing when the rays are not strictly in the plane of the singularity, being shot with the same values of the deviation angle $\theta$, but in the azimuth
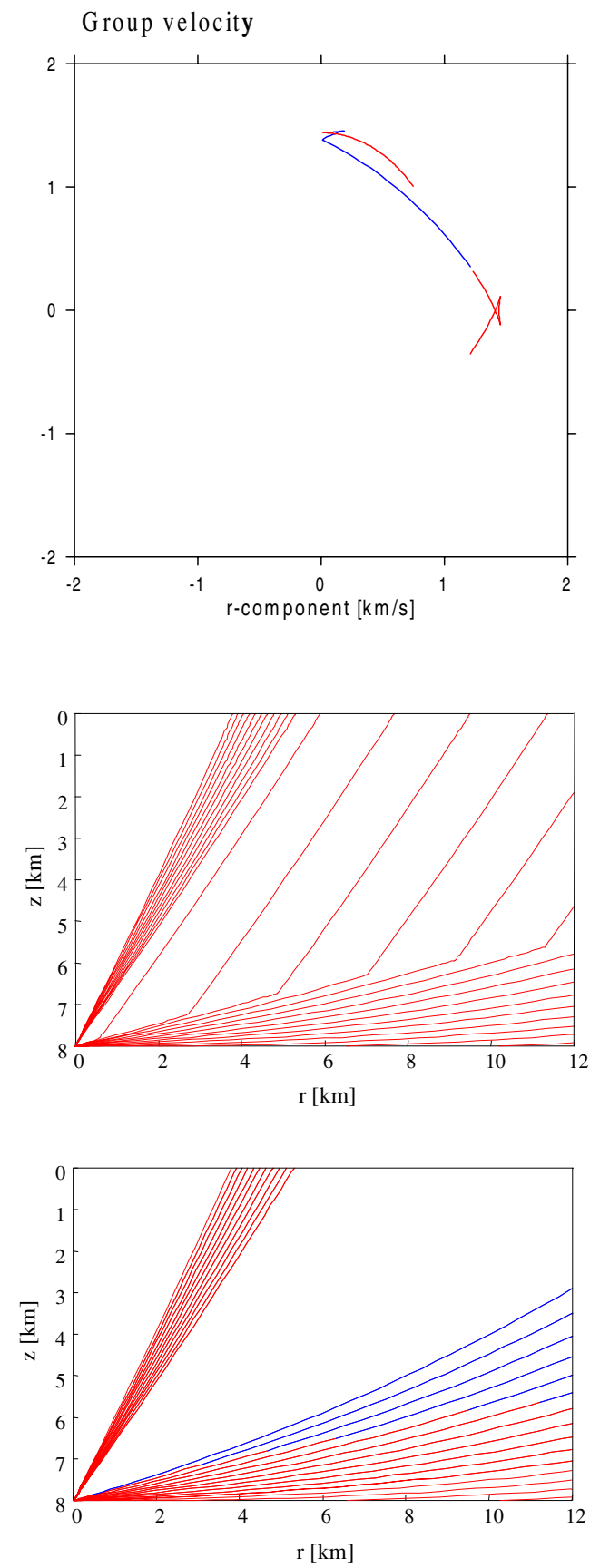


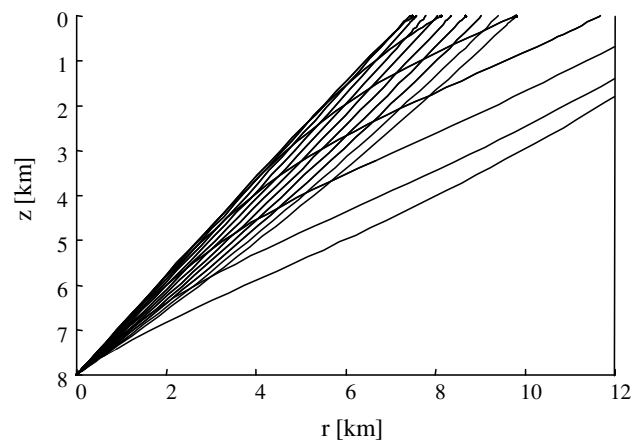

$\phi=47^{\circ}$. In this case, the ray cannot touch the singularity and the wave type ( $S 1$ or $S 2$ ) remains unchanged along the entire ray. Since the curvature of the phase velocity sheet is very high near the singularity, the ray can be bent significantly even if the velocity gradient in the medium is very weak. This situation also requires the polarization vectors to be calculated with high accuracy because of the extreme sensitivity of the ray direction to the polarization in this particular region. However, this bending occurs only in strongly anisotropic media and is limited

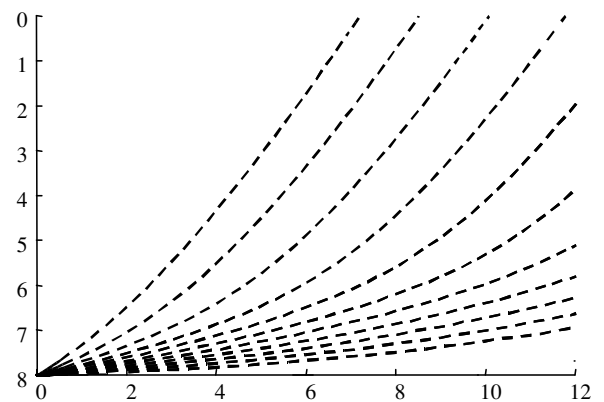

to the close vicinity of the conical point. Moreover, the ray bending is always smooth (see Fig. 10, lower plots). For weakly anisotropic media, this effect disappears.

\section{CONCLUSIONS}

We have proposed and tested a strategy for ray tracing in anisotropic inhomogeneous media that is applicable even in the singular directions and their vicinities. This ray tracing is 
numerically stable and yields correct results for all the following difficult situations: transition between isotropy and anisotropy, very weak anisotropy and all kinds of singularities in strong anisotropy.

The proposed approach is a slight modification of the ray tracing based on evaluating the right-hand sides of the equations by means of the polarization vector of the traced wave. The modification consists of imposing an additional condition requiring the polarization vector of the traced wave to be continuous along a ray. This condition is automatically satisfied when tracing rays in regular directions, but must be explicitly required in singular directions. If we do not treat the polarization vector in this way, the ray tracing can produce abrupt changes of the direction of the ray in the singularity. This abrupt bending of a ray, however, is unphysical in smoothly varying inhomogeneous media. The incorrectness of this bending is clear for simple types of singularity such as an intersection singularity, but less obvious for more complex singularities such as a conical point. The conical point displays a complicated geometry in both the phase and wave surfaces, and thus we cannot exclude a distinct distortion of rays by this singularity. This happens particularly in strong anisotropy, when the ray approaches the conical point passing it without touching. Although the rays can display a substantial bending in this case, this bending must always be smooth and the polarization vector of the traced wave must be continuous along a ray.

We also observe that the ray can split in the singularity. This appears if the symmetry of anisotropy beyond the singularity is different from that in front of the singularity. This is the case of smooth transitions between isotropy and anisotropy. It can also be observed under very weak anisotropy, where a small perturbation of elastic parameters can lead to a rapid change of the orientation of anisotropy, or to a change of anisotropy itself. As regards strong anisotropy, splitting in the singularity is probably not a frequent occurrence. Finally, we stress that whenever we observe the splitting of rays in the singularity in smoothly inhomogeneous anisotropic media, the splitting must be smooth, meaning that the split rays must have the same tangent in the singularity.

\section{ACKNOWLEDGMENTS}

I thank I. Pšenčík for critically reading the manuscript and for his comments. This work was performed at CPGG/UFBA, Salvador, Brazil, where the author was a visiting professor. The work was supported by the agencies CNPq and PGS of Brazil, by the Consortium Project 'Seismic waves in complex 3-D structures', and by the Grant Agency of the Czech Republic, grant no. 205/00/1350.

\section{REFERENCES}

Alshits, V.I. \& Lothe, E., 1979. Elastic waves in triclinic crystals. Topology of polarization fields and some general theorems, Crystallography, 24, 683-693 (in Russian).

Alshits, V.I., Sarychev, A.V. \& Shuvalov, A.L., 1985. Classification of the degenerations and analysis of their stability in elastic wave theory in crystals, J. Exper. Theor. Phys., 89, 922-938 (in Russian).

Babich, V.M., 1961. Ray method of calculating the intensity of wavefronts in the case of a heterogeneous, anisotropic, elastic medium, in Geophys. J. Int., 1994, 110, 379-383.
Burridge, R., 1967. The singularity on the plane lids of the wave surface of elastic media with cubic symmetry, Q. J. Mech. appl. Math., 20, $41-56$.

Červený, V., 1972. Seismic rays and ray intensities in inhomogeneous anisotropic media, Geophys. J. R. astr. Soc., 29, 1-13.

Červený, 2001. Seismic Ray Theory, Cambridge University Press, Cambridge, in press.

Červený, V. \& Firbas, P., 1984. Numerical modelling and inversion of travel times of seismic body waves in inhomogeneous anisotropic media, Geophys. J. R. astr. Soc., 76, 41-51.

Červený, V., Molotkov, I.A. \& Pšenčík, I., 1977. Ray Method in Seismology, Charles University Press, Praha.

Crampin, S. \& Yedlin, M., 1981. Shear-wave singularities of wave propagation in anisotropic media, J. Geophys., 49, 43-46.

Farra, V., 1989. Ray perturbation theory for heterogeneous hexagonal anisotropic media, Geophys. J. Int., 99, 723-737.

Gajewski, D. \& Pšenčík, I., 1987. Computation of high-frequency seismic wavefields in 3-D laterally inhomogeneous anisotropic media, Geophys. J. R. astr. Soc., 91, 383-411.

Gajewski, D. \& Pšenčík, I., 1990. Vertical seismic profile synthetics by dynamic ray tracing in laterally varying layered anisotropic structures, J. geophys. Res., 95, 11 301-11 315.

Grechka, V.Yu. \& \& Obolentseva, I.R., 1993. Geometrical structure of shear wave surfaces near singularity directions in anisotropic media, Geophys. J. Int., 115, 609-616.

Helbig, K., 1994. Foundations of Anisotropy for Exploration Seismics, Pergamon, New York.

Miller, G.F. \& Musgrave, M.J.P., 1956. On the propagation of elastic waves in aelotropic media, III, Media of cubic symmetry, Proc. $R$. Soc. Lond., A236, 352-383.

Musgrave, M.J.P., 1985. Acoustic axes in orthorhombic media, Proc. R. Soc. Lond., A401, 131-143.

Nowack, R.I. \& Pšenčík, I., 1991. Perturbation from isotropic to anisotropic heterogeneous media in the ray approximation, Geophys. J. Int., 106, 1-10.

Payton, R.G., 1992. Wave propagation in a restricted transversely isotropic elastic solid whose slowness surface contains conical points, Q. J. Mech. appl. Math., 45, 183-197.

Pšenčík, I. \& Dellinger, J., 2001. Quasi-shear waves in inhomogeneous weakly anisotropic media by the quasi-isotropic approach: a model study, Geophysics, 66, 308-319.

Rümpker, G. \& Thomson, C.J., 1994. Seismic-waveform effects of conical points in gradually varying anisotropic media, Geophys. J. Int., 118, 759-780.

Shearer, P.M. \& Chapman, C.H., 1989. Ray tracing in azimuthally anisotropic media-I. Results for models of aligned cracks in the upper crust, Geophys. J. Int., 96, 51-64.

Vavryčuk, V., 1997. Elastodynamic and elastostatic Green tensors for homogeneous weak transversely isotropic media, Geophys. J. Int., 130, 786-800.

Vavryčuk, V., 1999. Properties of $S$ waves near a kiss singularity: a comparison of exact and ray solutions, Geophys. J. Int., 138, $581-589$.

\section{APPENDIX A: SENSITIVITY OF RAY- TRACING EQUATIONS FOR $S$ WAVES IN WEAKLY ANISOTROPIC MEDIA TO POLARIZATION VECTORS}

In this Appendix we examine the sensitivity of eqs (7) to perturbations of polarization vectors when tracing rays of $S$ waves in weakly anisotropic media. We deal with the $S 1$ wave only, because the problem for the $S 2$ wave is analogous. We assume that the perturbation of the polarization vector $\Delta \mathbf{g}^{S 1}$ lies in the plane of the $S$-wave polarization and represents an error in determining the polarization vector $\mathbf{g}^{S 1}$ in a nearly 
degenerate medium produced by a numerical algorithm. The first equation in (7) is then expressed as

$\frac{d x_{i}}{d \tau}=a_{i j k l} p_{l}\left(g_{j}^{S 1}+\Delta g_{j}^{S 1}\right)\left(g_{k}^{S 1}+\Delta g_{k}^{S 1}\right)$.

Since $\Delta \mathbf{g}^{S 1}$ lies in the plane of the $S$-wave polarization, we can write

$\Delta g_{k}^{S 1}=\varepsilon g_{k}^{S 2}$,

where $\varepsilon$ is a small parameter. Hence,

$$
\begin{aligned}
\frac{d x_{i}}{d \tau} & =a_{i j k l} p_{l}\left(g_{j}^{S 1}+\varepsilon g_{j}^{S 2}\right)\left(g_{k}^{S 1}+\varepsilon g_{k}^{S 2}\right) \\
& \cong a_{i j k l} p_{l} g_{j}^{S 1} g_{k}^{S 1}+\varepsilon a_{i j k l} p_{l}\left(g_{j}^{S 1} g_{k}^{S 2}+g_{j}^{S 2} g_{k}^{S 1}\right),
\end{aligned}
$$

where we neglect the second-order perturbations. The first term in eq. (A3) is the exact right-hand side of the ray-tracing equation; the second term corresponds to the error due to an inaccurate polarization vector. Since this term is small, we can substitute the elasticity tensor $a_{i j k l}$ and slowness vector $p_{l}$ for weakly anisotropic media by relevant quantities for the isotropic background $a_{i j k l}^{0}$ and $p_{l}^{0}$. Moreover, we utilize the well-known identity for isotropic media,

$a_{i j k l}^{0}=\frac{\lambda}{\rho} \delta_{i j} \delta_{k l}+\frac{\mu}{\rho}\left(\delta_{i k} \delta_{j l}+\delta_{j k} \delta_{i l}\right)$.

We then obtain

$$
\begin{aligned}
& \varepsilon a_{i j k l} p_{l}\left(g_{j}^{S 1} g_{k}^{S 2}+g_{j}^{S 2} g_{k}^{S 1}\right) \\
& \simeq \varepsilon a_{i j k l}^{0} p_{l}^{0}\left(g_{j}^{S 1} g_{k}^{S 2}+g_{j}^{S 2} g_{k}^{S 1}\right) \\
& \quad=\frac{\varepsilon}{\rho}\left[(\lambda+\mu)\left(g_{i}^{S 1} p_{k}^{0} g_{k}^{S 2}+g_{i}^{S 2} p_{k}^{0} g_{k}^{S 1}\right)+2 \mu p_{i}^{0} g_{k}^{S 1} g_{k}^{S 2}\right]=0,
\end{aligned}
$$

where we used

$g_{k}^{S 1} g_{k}^{S 2}=0, \quad g_{k}^{S 1} p_{k}^{0} \cong 0 \quad$ and $\quad g_{k}^{S 2} p_{k}^{0} \cong 0$.

Symbol $\cong$ in eqs (A3), (A5) and (A6) means equality in the sense of the first-order perturbation theory.

Eq. (A5) proves that errors in the $S 1$-wave polarization vector affect the right-hand side of the first ray-tracing equation in (7) by a perturbation of second order only. The same result can also be obtained for the other equation in (7). 